\title{
A multicenter phase II study of TAS-114 in combination with S-1 in patients with pretreated advanced gastric cancer (EPOC1604)
}

\author{
Akihito Kawazoe $^{1}$ - Daisuke Takahari ${ }^{2}$. Chin Keisho ${ }^{2} \cdot$ Yoshiaki Nakamura $^{1} \cdot$ Takashi Ikeno $^{3}$. Masashi Wakabayashi ${ }^{3}$. \\ Shogo Nomura ${ }^{3} \cdot$ Hitomi Tamura $^{3} \cdot$ Miki Fukutani $^{3} \cdot$ Nami Hirano $^{3} \cdot$ Yumiko Saito $^{4} \cdot$ Moe Kambe $^{4}$ - Akihiro Sato ${ }^{3}$. \\ Kohei Shitara ${ }^{1}[$
}

Received: 9 May 2020 / Accepted: 13 July 2020 / Published online: 22 July 2020

(c) The International Gastric Cancer Association and The Japanese Gastric Cancer Association 2020

\begin{abstract}
Background This is a phase 2 study aimed at evaluating the efficacy and safety of TAS-114, a novel deoxyuridine triphosphatase inhibitor, combined with S-1 in patients with advanced gastric cancer (AGC).

Methods Eligible patients had AGC with measurable lesions, according to the Response Evaluation Criteria in Solid Tumors (RECIST, v1.1), with two or more previous chemotherapy regimens including fluoropyrimidines, platinum agents, and taxanes or irinotecan. The primary endpoint was objective response rate (ORR) according to the RECIST, v1.1. Twenty-nine patients were required according to Simon's optimal two-stage design, with one-sided $a=5 \%$ and power $=80 \%$. Threshold and expected ORRs were 5\% and 25\%. Patients received TAS-114 (400 mg/body, twice a day) and S-1 (30 mg/m², twice a day) for 14 days, followed by 7 days of rest in one 3-week cycle. Protein expression levels of dUTPase and BRCA1 in tumor samples were determined by immunohistochemistry.

Results Accrual was terminated in June 2018 because meeting the predefined efficacy criteria was considered difficult. ORR and disease control rate were 5.0\% [95\% confidence interval (CI), 0.1-24.9\%] and 70.0\% (95\% CI, 45.7-88.1\%), respectively, for all 20 patients enrolled. Median progression-free survival (PFS) and overall survival were 2.4 months (95\% CI, 1.2-3.3 months) and 7.1 months (95\% CI, 5.2-9.4 months), respectively. Median PFS in the groups with high and low dUTPase protein expression in the cytoplasm was 2.8 months (95\% CI, 1.4-3.9) and 1.6 months (95\% CI, 0.6-2.4), respectively [hazard ratio, 0.40 (95\% CI, 0.16-1.04), log-rank test two-sided $p=0.047$ ]. Grade 3 or higher treatment-related adverse events included anemia (20\%), leucopenia (15\%), neutropenia (10\%), rash (10\%), thrombocytopenia (5\%), and lymphopenia (5\%) Conclusions TAS-114 with S-1 showed only modest antitumor activity with acceptable safety profiles for patients heavily pretreated with AGC.
\end{abstract}

Keywords Advanced gastric cancer $\cdot$ TAS-114 $\cdot$ S-1

Electronic supplementary material The online version of this article (https://doi.org/10.1007/s10120-020-01107-y) contains supplementary material, which is available to authorized users.

Kohei Shitara

kshitara@east.ncc.go.jp

1 Department of Gastroenterology and Gastrointestinal Oncology, National Cancer Center Hospital East, 6-5-1 Kashiwanoha, Kashiwa, Chiba 277-8577, Japan

2 Department of Gastroenterology, The Cancer Institute Hospital of the Japanese Foundation for Cancer Research, Tokyo, Japan

\section{Introduction}

Gastric cancer is the fifth most common type of cancer and the third leading cause of death from cancer worldwide [1]. Patients with advanced gastric cancer (AGC) present a poor

3 Clinical Research Support Office, National Cancer Center Hospital East, Kashiwa, Chiba, Japan

4 Department of Clinical Research Department, The Cancer Institute Hospital of the Japanese Foundation for Cancer Research, Tokyo, Japan 
prognosis and are usually treated with systematic chemotherapy, including a combination of platinum and fluoropyrimidine [5-fluorouracil (5-FU), S-1, or capecitabine], trastuzumab (for HER2-positive cases), taxanes, irinotecan, and ramucirumab [2-6]. Two anti-PD-1 inhibitors have also been approved for treating AGC-pembrolizumab by the U.S. Food and Drug Administration for PD-L1-positive tumors and nivolumab in Asian countries, regardless of PD-L1 status [7, 8]. Most recently, trifluridine/tipiracil showed a survival benefit in heavily pretreated AGC patients [9]. Despite the recent increase in treatment options, median overall survival time of patients with AGC treated with a combination and sequential regimen involving these agents was less than 15 months. Therefore, the development of novel anticancer agents for patients with AGC is urgently required.

Deoxyuridine triphosphatase (dUTPase) is a gatekeeper enzyme that prevents uracil and 5-FU from misincorporating into DNA [10]. High expression of dUTPase in tumor tissues has been associated with resistance to 5-FU-based chemotherapy [11]. TAS-114 is a potent dUTPase inhibitor, which prevents dUTP and 5-fluoro-dUTP (FdUTP) conversion into their respective monophosphate forms. Although TAS-114 itself does not show antitumor activity, it can increase the amount of FdUTP in the tumor for selective incorporation into DNA. This enhances antitumor activity of 5-FU or fluoropyrimidines, such as $\mathrm{S}-1$ or capecitabine, compared with 5-FU alone in various human cancer xenograft models [12, 13]. Furthermore, a phase 1 study of TAS-114 in combination with S-1 in Japanese patients showed its tolerability and preliminary antitumor signals for patients with non-small cell lung cancer (NSCLC) and AGC [14].

Based on these results, we conducted a phase 2 study to assess the efficacy and safety of a combination of TAS-114 and $\mathrm{S}-1$ in patients with AGC.

\section{Methods}

\section{Study design and participants}

This study is a multicenter phase 2 trial to evaluate the use of TAS-114 in combination with S-1 in AGC patients in Japan. Criteria for patient enrolment included (1) histologically or cytologically confirmed advanced or recurrent gastric cancer, (2) an Eastern Cooperative Oncology Group performance status of 0 or 1, (3) a history of two or more previous chemotherapy regimens containing fluoropyrimidine, platinum agents, and taxanes or irinotecan, (4) measurable lesions specified in the Response Evaluation Criteria in Solid Tumors (RECIST, v1.1), (5) an adequate bone marrow reserve, and (6) adequate hepatic and renal function. Major exclusion criteria included (1) a history of previous treatment with TAS-114 and (2) the presence of a serious comorbidity. A full list of inclusion and exclusion criteria is included in the protocol.

All patients provided written informed consent prior to participating in the study. The study was conducted according to the Declaration of Helsinki and Good Clinical Practice Guidelines, following approval by the Ethics Board of each institution. The study protocol is listed in the UMIN registry (UMIN000028329).

\section{Procedures}

Eligible patients received TAS-114 (400 mg/body, twice a day) and S-1 (30 mg/m², twice a day) for 14 days, followed by 7 days of rest in one 3-week cycle until disease progression, development of intolerable toxicity, or withdrawal of consent, with one cycle lasting 3 weeks [14, 15]. Body surface area (BSA)-based TAS-114 dosing was changed to a flat-fixed dose to avoid the risk of dosing errors. A dose of $400 \mathrm{mg}$ of TAS-114 was calculated based on a mean BSA of $1.69 \mathrm{~m}^{2}$ obtained in the two phase 1 studies [14, 15]. Toxicity was managed with supportive care, prespecified reductions in TAS-114 and S-1 doses, and interruptions of study drug doses according to the protocol. If a dose was lowered, it could not be increased in subsequent cycles. Dose reduction, interruption, and discontinuation had to be implemented simultaneously for TAS-114 and S-1. Study treatment was discontinued if the subsequent cycle was delayed for more than 3 weeks. Dose modifications of study drugs are described in detail in the protocol.

Tumor response was evaluated according to the RECIST, v1.1, using CT or MRI at baseline and every 6 weeks until disease progression or the beginning of subsequent treatment. Adverse events were evaluated throughout the treatment period using the National Cancer Institute Common Terminology Criteria for Adverse Events (version 4.03). According to the concept of TAS114 , dUTPase is one of the expected biomarkers for this combination. Also, a phase 1 trial of TAS-114 plus S-1 showed that low breast cancer 1 and early-onset (BRCA1) level may be more sensitive to TAS-114 with S-1 than that with high BRCA1 level [14]. Protein expression levels of dUTPase and BRCA1 in formalin fixed paraffin embedded tumor specimens were determined by immunohistochemistry by SRL (Tokyo, Japan), as described previously [14]. Median protein expression level was used for the high vs. low cut-off value.

The data center of the Clinical Research Support Office at the National Cancer Centre Hospital East, Japan (NCCHE-OCRS) confirmed patient eligibility. Data were collected, analyzed, and interpreted at the NCCHE-OCRS (study number: EPOC 1604). 


\section{Outcomes}

As assessed according to the RECIST, v1.1, the primary endpoint was objective response rate (ORR), and the secondary endpoints included incidence of adverse events, disease control rate (DCR), progression-free survival (PFS), and overall survival (OS). ORR was defined as the proportion of patients with the best overall complete response (CR) or partial response (PR), and DCR was the proportion of patients with the best overall response of CR, PR, or stable disease (SD). PFS was the time from the date of registration until the date of disease progression or death from any cause, whichever occurred first, and OS was the time from the date of registration until the date of death from any cause. The exploratory endpoint included biomarker analysis such as dUTPase and BRCA1 expressions in archival tumor tissues. The association between expression levels and efficacy of the study treatment was investigated.

\section{Statistical analyses}

The planned sample size was 29 patients based on Simon's optimal two-stage design with one-sided $a=5 \%$ and power $=80 \%$ [16]. Threshold and expected ORRs were 5\% and $20 \%$, respectively. Ten patients were enrolled in the first stage and if one or more patients achieved confirmed objective response (CR or PR), 19 more were planned to be enrolled in the second stage. Otherwise, the study would not have proceeded to the second stage, and we would have considered discontinuing the trial. The primary endpoint would be met if four or more patients out of 29 achieved objective response.

Patient characteristics, safety data, and antitumor activity were summarized descriptively. ORR and DCR confidence intervals (CIs) were constructed using the Clopper-Pearson method, and PFS and OS were estimated using the Kaplan-Meier method. All statistical analyses for the efficacy endpoints were performed on a patient set who was eligible and received protocol treatment at least once. The safety endpoints for patients who received protocol treatment at least once were analyzed. Statistical analyses were performed using the SAS Release version 9.4 statistical software suite (SAS Institute, Cary, NC, USA).

\section{Results}

\section{Patients}

One patient achieved confirmed objective response out of the 10 patients enrolled in the first stage between October 17, 2017, and December 12, 2017. Therefore, we started the second stage on February 22, 2018. None of the first 10 patients enrolled in the second stage achieved objective response. At the same time, it was reported that a randomized, international phase 2 trial of TAS- 114 plus S- 1 vs. S- 1 monotherapy in 5-FU-naïve patients with NSCLC failed to show antitumor activity and presented increased incidences of adverse events [17]. Accrual was terminated in June 2018 based on these results. A total of 20 patients were enrolled in this study between October 17, 2017, and June 14, 2018 (Table 1). Median age was 65 years (range, 34-77 years), and 18 patients $(90 \%)$ were male. ECOG performance status was 0 in $75 \%$ of patients. Fifteen patients $(75 \%)$ had received three or more previous chemotherapy regimens. All patients had been previously treated with fluoropyrimidines (14 patients with S-1), platinum agents, and taxane agents.

Data cut-off for safety and efficacy analysis was on October 31, 2018, with a median follow-up of 6.5 months (range, 1.7-11.6 months). The median number of treatment cycles was 3 (range, 1-8 cycles). No patients were undergoing

Table 1 Baseline characteristics

\begin{tabular}{|c|c|}
\hline Characteristics & $\begin{array}{l}\text { All patients } \\
(n=20)\end{array}$ \\
\hline \multicolumn{2}{|l|}{ Age, years } \\
\hline Median (range) & $65(34-77)$ \\
\hline \multicolumn{2}{|l|}{ Sex } \\
\hline Male & $18(90 \%)$ \\
\hline Female & $2(10 \%)$ \\
\hline \multicolumn{2}{|l|}{ ECOG PS } \\
\hline 0 & $15(75 \%)$ \\
\hline 1 & $5(25 \%)$ \\
\hline \multicolumn{2}{|l|}{ Primary site } \\
\hline Gastric & $19(95 \%)$ \\
\hline Gastroesophageal junction & $1(5 \%)$ \\
\hline \multicolumn{2}{|l|}{ Histology } \\
\hline Diffuse & $9(45 \%)$ \\
\hline Intestinal & $11(55 \%)$ \\
\hline \multicolumn{2}{|l|}{ Site of metastases } \\
\hline Liver & $8(40 \%)$ \\
\hline Peritoneum & $5(25 \%)$ \\
\hline Prior gastrectomy & $9(45 \%)$ \\
\hline \multicolumn{2}{|c|}{ Number of prior chemotherapies } \\
\hline 2 & $5(25 \%)$ \\
\hline 3 or more & $15(75 \%)$ \\
\hline \multicolumn{2}{|l|}{ Prior regimens } \\
\hline Fluoropyrimidines & $20(100 \%)$ \\
\hline Platinum agents & $20(100 \%)$ \\
\hline Taxane agents & $20(100 \%)$ \\
\hline Irinotecan & $10(50 \%)$ \\
\hline HER2 positive & $5(25 \%)$ \\
\hline
\end{tabular}

ECOG eastern cooperative oncology group, HER-2 human epidermal growth factor receptor 2 


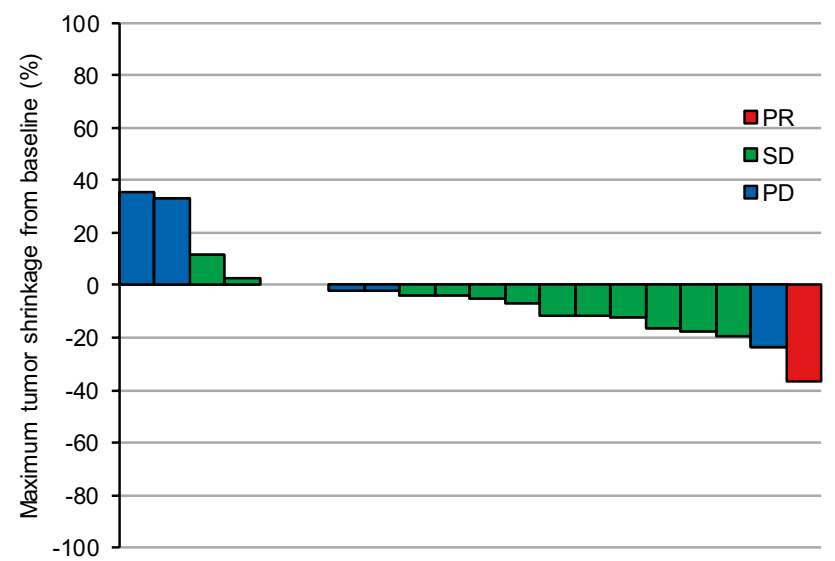

Fig. 1 Waterfall plot of maximum percent change in tumor size from baseline measured according to the response evaluation criteria in solid tumor (RECIST). $C R$ complete response, $P R$ partial response, $S D$ stable disease, $P D$ progressive disease, $M M R$ mismatch repair

Table 2 Tumor responses

\begin{tabular}{ll}
\hline & All patients $(n=20)$ \\
\hline Complete response & 0 \\
Partial response & 1 \\
Stable disease & 13 \\
Disease progression & 6 \\
Objective response rate & $5.0 \%(95 \% \mathrm{CI}, 0.1-24.9)$ \\
Disease control rate & $70.0 \%(95 \% \mathrm{CI}, 45.7-88.1)$ \\
\hline
\end{tabular}

treatment at data cut-off, and 20 patients (100\%) had discontinued the protocol treatment due to disease progression $(n=18)$ or patient refusal $(n=2)$. Fourteen patients received post-chemotherapies including nivolumab $(n=5)$, irinotecan $(n=5)$, S-1 plus oxaliplatin $(n=1)$, and study drugs $(n=3)$ after protocol treatment.

\section{Efficacy}

Objective response was observed in one patient, resulting in an ORR of 5\% (95\% CI, 0.1-24.9) in the entire population (Fig. 1 and Table 2). DCR was 70\% (95\% CI, 45.7-88.1) (Table 2), and 10 out of 13 patients with SD showed a decrease in tumor size from baseline (Fig. 1). ORR and DCR were $0 \%$ (95\% CI, 0.0-23.2) and 57.1\% (95\% CI, 28.9-82.3) in patients previously treated with S-1, and $16.7 \%(95 \%$ CI, 0.4-64.1) and 100\% (95\% CI, 54.1-100) in previously untreated patients, respectively.

Median PFS was 2.4 months (95\% CI, 1.2-3.3 months) (Fig. 2a). No patients achieved PFS at 6 months. Median PFS was 2.1 months (95\% CI, 1.2-2.8) in patients previously treated with S-1 and 3.1 months (95\% CI, 1.1-4.2) in previously untreated patients. Eight patients were still alive at data cut off and median OS was 7.1 months (95\% CI, 5.2-9.4 months) (Fig. 2b).

\section{Safety}

Treatment-related adverse events of any grade occurred in 18 patients (90\%), and common events were rash (55\%), anemia (40\%), decreased appetite (30\%), neutropenia (25\%), and thrombocytopenia (20\%) (Table 3). Grade 3 or higher treatment-related adverse events occurred in nine patients $(45 \%)$, including anemia (20\%), leucopenia $(15 \%)$, neutropenia (10\%), rash (10\%), thrombocytopenia (5\%), and lymphopenia (5\%). Most of these events were reversible through dose reduction or study drug interruption. Severe adverse events were observed in three patients (15\%), including grade 4 seizure, grade 2 fever, and grade 5 small intestinal perforation. Grade 2 fever was judged to be related to the study treatment by the investigator, while the others were not. Grade 5 small intestinal perforation was due to disease progression of peritoneal metastasis observed by the investigator. No treatment-related deaths occurred, and there were no treatment-related adverse events that led to study discontinuation. Treatment-related adverse events led to dose interruptions in 15 patients (75\%) and at least one dose reduction of the study drugs in 8 patients (40\%); one-level dose reduction in 6 patients and two-level reductions in 2 patients. Most dose reductions were due to rash, decreased appetite, or fatigue. Ten $(50 \%)$ patients experienced dose delay due to treatmentrelated adverse events.

\section{Biomarkers}

dUTPase and BRCA1 expression could be evaluated in all patients (Online Resource 1 and Online Resource 2). DCR in groups with high $(n=10)$ and low $(n=10)$ dUTPase protein expression in the cytoplasm was $80.0 \%$ (95\% CI, 44.4-97.5) and $60.0 \%$ (95\% CI, 26.2-87.8), respectively. Median PFS in groups with high $(n=10)$ and low $(n=10)$ dUTPase protein expression in the cytoplasm was 2.8 months $(95 \%$ CI, 1.4-3.9) and 1.6 months (95\% CI, 0.6-2.4), respectively [hazard ratio, 0.40 (95\% CI, 0.16-1.04), log-rank test $p=0.047$ ] (Online Resource 3a). The patient with objective response had high dUTPase expression. DCR in groups with high $(n=10)$ and low $(n=10)$ BRCA1 protein expression in the nucleus was $60.0 \%$ (95\% CI, 26.2-87.8) and 80.0\% (95\% CI, 44.4-97.5), respectively. Median PFS in groups with high $(n=10)$ and low $(n=10)$ BRCA1 protein expression in the nucleus was 2.3 months (95\% CI, 1.2-2.8) and 2.6 months (95\% CI, 0.6-3.9), respectively [hazard ratio, 0.94 (95\% CI, 0.38-2.33), $p=0.926$ ] (Online Resource 3b). 
Fig. 2 a Kaplan-Meier plots of progression-free survival (PFS). Median progression-free survival (PFS) was 2.4 months. b Overall survival (OS). Median OS was 7.1 months

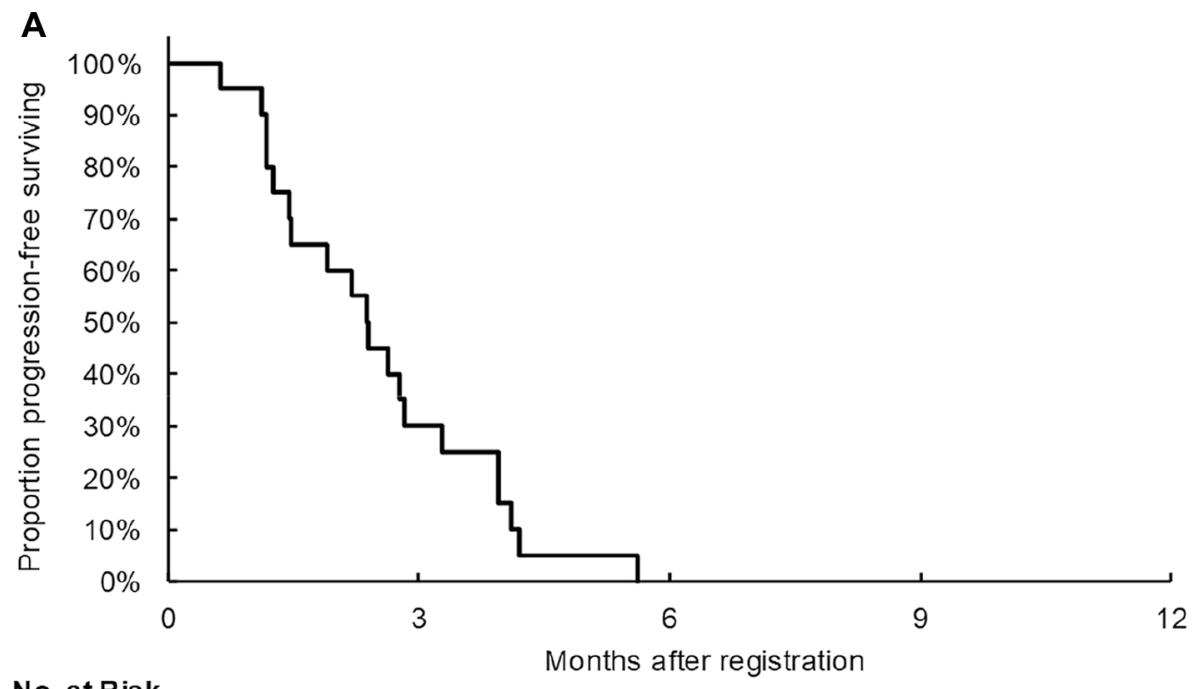

No. at Risk

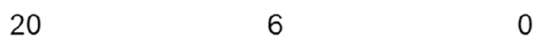

B

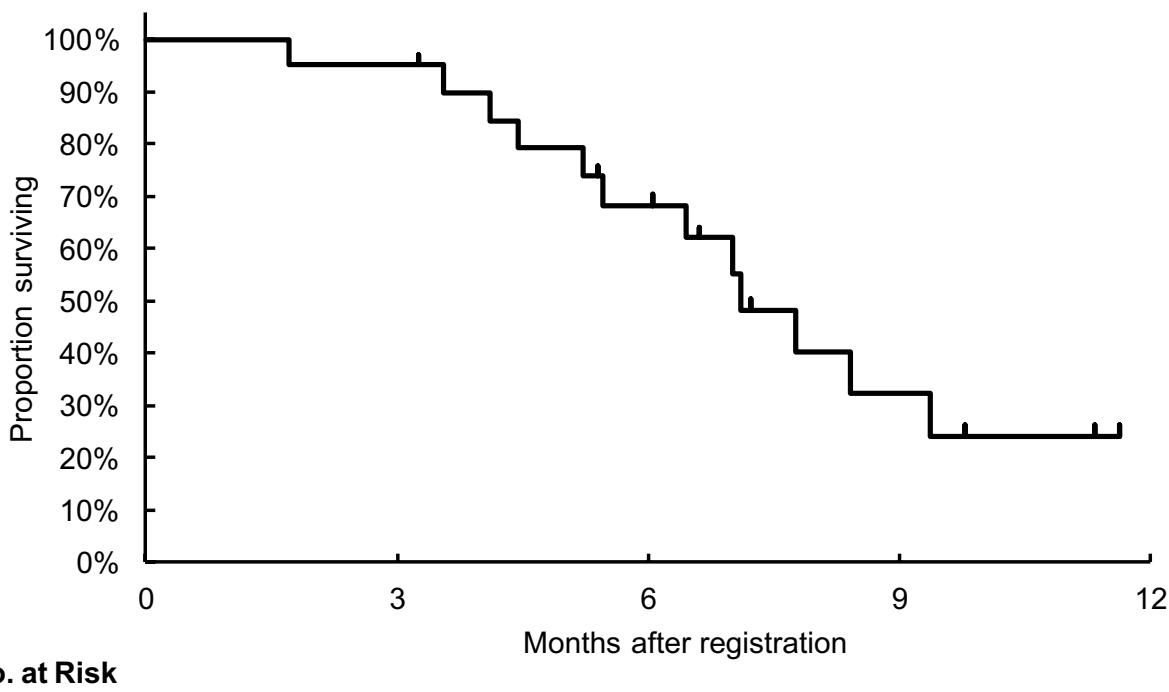

20

19

4

0

\section{Discussion}

Our trial demonstrated that TAS-114 with S-1 had only modest antitumor activity for patients heavily pretreated with AGC. To the best of our knowledge, this is the first phase 2 study that has evaluated the combined activity of TAS-114 and S-1 in AGC patients. Most treatment-related adverse events were manageable with dose reductions or interruptions, or with supportive care.

In this study, TAS-114 with S-1 provided an ORR of 5\% and a DCR of $70 \%$ with median PFS of 2.4 months and median OS of 7.1 months for AGC patients in third- or later line settings. These clinical outcomes seem to be comparable to those of the phase 3 study on trifluridine/tipiracil for AGC patients in the same setting [9], although no patients achieved PFS at 6 months in this study. Considering that all patients were previously treated with fluoropyrimidines in this study, dUTPase inhibition by TAS-114 in combination with S-1 might show some clinical benefits for 5-FU refractory patients, although modest activity of S-1 monotherapy after failure of platinum plus 5-FU was also observed in metastatic esophageal carcinoma [18-20]. However, only one out of the 20 patients achieved an objective response, which was considered to be difficult to meet predefined criteria of efficacy. Also, the phase 2 trial of TAS-114 plus S- 1 in patients with NSCLC failed to show antitumor activity 
Table 3 Treatment-related adverse events $(\geq 10 \%)$

\begin{tabular}{lcl}
\hline Adverse event & $n=20$ & \\
\cline { 2 - 3 } & All grades & Grade $\geq 3$ \\
\hline All events & $18(90 \%)$ & $9(45 \%)$ \\
Rash & $11(55 \%)$ & $2(10 \%)$ \\
Anemia & $8(40 \%)$ & $4(20 \%)$ \\
Decreased appetite & $6(30 \%)$ & $0(0 \%)$ \\
Neutropenia & $5(25 \%)$ & $2(10 \%)$ \\
Thrombocytopenia & $4(20 \%)$ & $1(5 \%)$ \\
Leucopenia & $3(15 \%)$ & $3(15 \%)$ \\
Nausea & $3(15 \%)$ & $0(0 \%)$ \\
Diarrhea & $2(10 \%)$ & $0(0 \%)$ \\
Mucositis & $2(10 \%)$ & $0(0 \%)$ \\
Fever & $2(10 \%)$ & $0(0 \%)$ \\
Pruritus & $2(10 \%)$ & $0(0 \%)$ \\
\hline
\end{tabular}

with increased incidence of toxicities [17]. We decided to discontinue the trial in the middle of the second stage based on these results. The lack of sufficient efficacy of this combination suggests that not only dUTPase but also other molecules or pathways may be associated with resistance to 5-FU-based chemotherapy. Indeed, a previous study showed that uracil DNA glycosylase (UGD) is also an important enzyme that prevents uracil and 5-FU from misincorporating into DNA. Also, UDG depletion significantly enhanced 5-FU cytotoxicity through replication fork collapse-induced DNA damage in cancer cell lines [21]. The precise mechanism of 5-FU resistance and novel treatment strategies to overcome it should be investigated in a future study.

Exploratory analysis showed that patients with high dUTPase expression had a longer PFS than those with low expression in this study, which might be consistent with the concept of targeting dUTPase using TAS-114 to overcome 5-FU resistance. On the contrary, BRCA1 expression was not associated with clinical outcomes in this study, which is not consistent with the previous phase 1 study [14]. A preclinical study showed that suppression of DNA repair proteins, such as BRCA1, involved in homologous recombination repair by siRNA in HeLa cells increased their sensitivity to TAS-114 combined with the 5-FU metabolite 5-fluoro-2-deoxyuridine [22]. The sample was not large enough to assess the correlation between these biomarkers and the efficacy of this combination.

The safety profiles in this study were almost consistent with previous phase 1 studies and the phase 2 study for NSCLC $[14,15,17]$. In the phase 2 study for NSCLC, the incidence rates of anemia, skin toxicities, and grade $\geq 3$ treatment-related adverse events were higher in the TAS114 and S-1 group than in the monotherapy group, although they were manageable through dose modification [17]. Common toxicities, such as rash, anemia, decreased appetite, neutropenia, and thrombocytopenia, reported in this study, were also manageable with dose reductions, dose interruptions, and supportive care. Although the precise mechanism is unknown, it is possible that the inhibition of dUTPase by TAS-114 on skin increased rash due to fluoropyrimidines. No patients discontinued the study treatment because of treatment-related adverse events. Only one serious treatment-related adverse event (grade 2 fever) occurred. No treatment-related deaths occurred.

This study had several limitations. It was not randomized as the phase 2 trial with small sample size only for Japanese patients; thus, any efficacy and safety results as well as biomarker assessment are preliminary in nature. In this study, $75 \%$ of patients had an ECOG PS of 0, suggesting that a selected population was enrolled in the trial, which might lead to a relatively longer OS in third- or later line settings. Moreover, clinical outcomes were not evaluated according to the prespecified study design since accrual was terminated due to the lack of sufficient efficacy in this trial and the other trial [17].

In conclusion, TAS-114 with S-1 showed modest antitumor activity with acceptable safety profiles for patients heavily pretreated with AGC, but did not show sufficient efficacy to proceed to the next step for the treatment of AGC.

Acknowledgements We thank all the patients and their families, physicians, research nurses, study coordinators, and research staff who participated in this study. Particularly, we acknowledge all the National Cancer Centre Exploratory Oncology Research and Clinical Trial Centre members for their work.

Funding This investigation was funded by TAIHO PHARMACEUTICAL CO., LTD.

\section{Compliance with ethical standards}

Conflict of interest AK reports grants and personal fees from Taiho, grants and personal fees from Ono, grants from Sumitomo Dainippon, and grants from MSD, outside the submitted work. DT reports grants and personal fees from Taiho Pharmaceutical Co. and Ono Pharmaceutical Co., and personal fees from Eli Lilly Japan, Bristol-Myers Squibb, Yakult Honsha Co., Ltd., and Chugai Pharmaceutical Co., Ltd., outside the submitted work. CK has nothing to disclose. YN reports research funding from Taiho Pharmaceutical. TI has nothing to disclose. MW reports personal fees from Chugai Pharmaceutical Co., Ltd. and personal fees from Johnson \& Johnson K.K. MEDICAL COMPANY, outside the submitted work. SN reports personal fees from AstraZeneca, personal fees from Taiho Pharmaceutical, personal fees from Chugai, and personal fees from Pfizer, outside the submitted work. HT has nothing to disclose. MF has nothing to disclose. NH has nothing to disclose. YS has nothing to disclose. MK has nothing to disclose. AS has nothing to disclose. KS reports receiving payment for consulting or advisory roles from Astellas, Lilly, Bristol-Myers Squibb, Takeda, Pfizer, Ono, and MSD; honoraria from Novartis, AbbVie, and Yakult; and research funding from Astellas, Lilly, Ono, Sumoitomo Dainippon, Daiichi Sankyo, Taiho, Chugai, MSD, and Medi Science.

Human rights statement All procedures followed were in accordance with the ethical standards of the responsible committee on human 
experimentation (institutional and national) and with the Helsinki Declaration of 1964 and later versions.

Informed consent Informed consent or substitute was obtained from all patients for being included in the study.

\section{References}

1. International Agency for Research on Cancer. Globocan 2018: stomach. https://gco.iarc.fr/today/data/factsheets/cancers/7-Stoma ch-fact-sheet.pdf. Accessed 4 Oct 2018.

2. Cunningham D, Starling N, Rao S, Iveson T, Nicolson M, Coxon $\mathrm{F}$, et al. Capecitabine and oxaliplatin for advanced esophagogastric cancer. N Engl J Med. 2008;358:36-46.

3. Koizumi W, Narahara H, Hara T, Takagane A, Akiya T, Takagi M, et al. S-1 plus cisplatin versus S-1 alone for first-line treatment of advanced gastric cancer (SPIRITS trial): a phase III trial. Lancet Oncol. 2008;9:215-21.

4. Bang YJ, Van Cutsem E, Feyereislova A, Takagane A, Akiya T, Takagi M, et al. Trastuzumab in combination with chemotherapy versus chemotherapy alone for treatment of HER2-positive advanced gastric or gastro-oesophageal junction cancer (ToGA): a phase 3, open-label, randomized controlled trial. Lancet. 2010;376:687-97.

5. Wilke H, Muro K, Van Cutsem E, Oh SC, Bodoky G, Shimada Y, et al. Ramucirumab plus paclitaxel versus placebo plus paclitaxel in patients with previously treated advanced gastric or gastrooesophageal junction adenocarcinoma (RAINBOW): a doubleblind, randomised phase 3 trial. Lancet Oncol. 2014;15:1224-355.

6. Kang JH, Lee SI, Lim Do H, Park KW, Oh SY, Kwon HC. Salvage chemotherapy for pretreated gastric cancer: a randomized phase III trial comparing chemotherapy plus best supportive care with best supportive care alone. J Clin Oncol. 2012;30:1513-8.

7. Fuchs CS, Doi T, Jang RW, Muro K, Satoh T, Machado M, et al. Safety and efficacy of pembrolizumab monotherapy in patients with previously treated advanced gastric and gastroesophageal junction cancer: phase 2 clinical KEYNOTE-059 trial. JAMA Oncol. 2018;4:e180013.

8. Kang YK, Boku N, Satoh T, Ryu MH, Chao Y, Kato K, et al. Nivolumab in patients with advanced gastric or gastro-oesophageal junction cancer refractory to, or intolerant of, at least two previous chemotherapy regimens (ONO-4538-12, ATTRAC TION-2): a randomised, doubleblind, placebo-controlled, phase 3 trial. Lancet. 2017;390:2461-71.

9. Shitara K, Doi T, Dvorkin M, Mansoor W, Arkenau HT, Prokharau A, et al. Trifluridine/tipiracil versus placebo in patients with heavily pretreated metastatic gastric cancer (TAGS): a randomised, double-blind, placebo-controlled, phase 3 trial. Lancet Oncol. 2018;19:1437-48.

10. Canman CE, Radany EH, Parsels LA, Davis MA, Lawrence TS, Maybaum J. Induction of resistance to fluorodeoxyuridine cytotoxicity and DNA damage in human tumor cells by expression of Escherichia coli deoxyuridine triphosphatase. Cancer Res. 1994;54:2296-8.

11. Ladner RD, Lynch FJ, Groshen S, Xiong YP, Sherrod A, Caradonna SJ, et al. dUTP nucleotidohydrolase isoform expression in normal and neoplastic tissues: association with survival and response to 5-fluorouracil in colorectal cancer. Cancer Res. 2000;60:3493-503.

12. Yano W, Yokogawa T, Wakasa T, Yamamura K, Fujioka A, Yoshisue K, et al. TAS-114, a first-in-class dual dUTPase/DPD inhibitor, demonstrates potential to improve therapeutic efficacy of fluoropyrimidine-based chemotherapy. Mol Cancer Ther. 2018;17:1683-93.

13. Yokogawa T, Wakasa T, Yano W, Yoshisue K, Fujioka A, Eshima $\mathrm{K}$, et al. TAS-114 enhances S-1 activity in vivo when used in combination. Eur J Cancer. 2012;48:22.

14. Doi T, Yoh K, Shitara K, Takahashi H, Ueno M, Kobayashi S, et al. First-in-human phase 1 study of novel dUTPase inhibitor TAS-114 in combination with S-1 in Japanese patients with advanced solid tumors. Invest New Drugs. 2019;37:507-18.

15. Fasolo A, Aoyama T, Stathis A, et al. A large phase I study of TAS-114 in combination with S-1 in patients with advanced solid tumors. Proceedings: AACR Annual Meeting 2018; 14-18 April 2018; Chicago, IL. Abstract \#CT014.

16. Kunz CU, Wason JM, Kieser M. Two-stage phase II oncology designs using short-term endpoints for early stopping. Stat Methods Med Res. 2017;26:1671-83.

17. Yamamoto N, Hayashi H, Planchard D, Morán T, Gregorc V, Dowell J, et al. A randomized, phase 2 study of deoxyuridine triphosphatase inhibitor, TAS-114, in combination with S-1 versus $\mathrm{S}-1$ alone in patients with advanced non-small-cell lung cancer. Invest New Drugs. 2020. https://doi.org/10.1007/s10637-02000930-5(Epub ahead of print)

18. Ito T, Honma Y, Hirano H, Shoji H, Okita N, Iwasa S, et al. S-1 monotherapy after failure of platinum plus 5-fluorouracil chemotherapy in recurrent or metastatic esophageal carcinoma. Anticancer Res. 2019;39:3931-6.

19. Yasui H, Yoshino T, Boku N, Onozawa Y, Hironaka S, Fukutomi A, et al. Retrospective analysis of S-1 monotherapy in patients with metastatic colorectal cancer after failure to fluoropyrimidine and irinotecan or to fluoropyrimidine, irinotecan and oxaliplatin. Jpn J Clin Oncol. 2009;39:315-20.

20. Ono A, Boku N, Onozawa Y, Hironaka S, Fukutomi A, Yasui $\mathrm{H}$, et al. Activity of S-1 in advanced or recurrent gastric cancer patients after failure of prior chemotherapy, including irinotecan + cisplatin or fluorouracil (except S-1). Jpn J Clin Oncol. 2009;39:332-5

21. Yan Y, Han X, Qing Y, Condie AG, Gorityala S, Yang S, et al. Inhibition of uracil DNA glycosylase sensitizes cancer cells to 5-fluorodeoxyuridine through replication fork collapse-induced DNA damage. Oncotarget. 2016;7:59299-313.

22. Tsukioka S, Yano W, Yokogawa T, Wakasa T, Fujioka A, Yamamura $\mathrm{K}$, et al. Expression of DNA damage repair enzymes determine the efficacy of a novel dUTPase inhibitor, TAS-114. Presented at the AACR-NCI-EORTC International Conference: molecular targets and cancer therapeutics, Boston, MA, USA, 19-23 October 2013

Publisher's Note Springer Nature remains neutral with regard to jurisdictional claims in published maps and institutional affiliations. 